\title{
Full-complex amplitude modulation with binary spatial light modulators
}

\author{
Erdem Ulusoy, * Levent Onural, and Haldun M. Ozaktas \\ Department of Electrical and Electronics Engineering, Bilkent University, TR-06800 Bilkent, Ankara, Turkey \\ ${ }^{*}$ Corresponding author: eulusoy@bilkent.edu.tr
}

Received June 27, 2011; revised September 23, 2011; accepted September 23, 2011; posted September 26, 2011 (Doc. ID 149942); published October 19, 2011

\begin{abstract}
Imperfections and nonrobust behavior of practical multilevel spatial light modulators (SLMs) degrade the performance of many proposed full-complex amplitude modulation schemes. We consider the use of more robust binary SLMs for this purpose. We propose a generic method, by which, out of $K$ binary (or 1 bit) SLMs of size $M \times N$, we effectively create a new $2^{K}$-level (or $K$ bit) SLM of size $M \times N$. The method is a generalization of the well-known concepts of bit plane representation and decomposition for ordinary gray scale digital images and relies on forming a properly weighted superposition of binary SLMs. When $K$ is sufficiently large, the effective SLM can be regarded as a full-complex one. Our method is as efficient as possible from an information theoretical perspective. A $4 f$ system is discussed as a possible optical implementation. This $4 f$ system also provides a means for eliminating the undesirable higher diffraction orders. The components of the $4 f$ system can easily be customized for different production technologies. (c) 2011 Optical Society of America
\end{abstract}

OCIS codes: $\quad 070.0070,090.0090,070.6120,090.1760,090.2870,100.2810$

\section{INTRODUCTION}

Spatial light modulators (SLMs) are dynamically programmable two-dimensional (2D) optical masks on which computergenerated holograms (CGHs) are written to synthesize light fields in a number of applications, including three-dimensional (3D) holographic video displays [1-4]. Since CGHs are, in general, complex valued, an SLM that provides full-complex modulation would be very convenient, but such an SLM currently does not exist in practice. Most practical SLMs perform only a restricted type of modulation on the incoming light, such as amplitude-only or phase-only modulation, and even that restricted modulation is imperfect. These restrictions and imperfections of SLMs need to be handled in light field synthesis applications.

One approach is to devise coding methods such that the ideal complex-valued CGH that generates a desired light field is encoded in a CGH that is suitable for writing on the physical SLM [5,6]. Many different encoding methods for multilevel amplitude-only, multilevel phase-only, and binary SLMs have been proposed [ $\underline{7}-15]$. In these methods, when the observation region is considered, the actual SLM behaves like a lower-resolution full-complex SLM. However, when such methods are used, the SLM generates side beams such as conjugate images, dc terms, or quantization-related noise terms in addition to the desired light field. Removal of these side beams is problematic, especially when many SLMs need to be placed side by side, as in 3D display applications.

Another approach is to effectively create full-complex SLMs out of restricted type SLMs. As for some examples, in one of the proposed methods, an amplitude-only SLM is imaged on a phase-only SLM [16]. In this manner, light passing through the SLMs is modulated both in phase and amplitude as if it comes out of a single full-complex SLM. In another method, the beams of two phase-only SLMs are added using a beam splitter [17]. In that way, effectively, a new SLM is created, where a pixel of the new SLM is equal to the sum of two phase-only pixels, so it can be adjusted to a large number of different complex values. Similar methods are discussed in [18-23]. These methods are successful in the sense that the new SLM provides a richer modulation compared to the component SLMs. However, a common problem in all these methods is that the set of complex values available for a pixel of the new SLM does not have a good coverage of the complex plane, so it is hard to regard the new SLM as a satisfactory full-complex SLM. The main reason of this problem is the imperfections of practical SLMs: for instance, most phase-only SLMs do not cover the $0-2 \pi$ range for the phase, but cover only a restricted angular range. Similarly, most phase-only SLMs perform an undesired amplitude modulation along with the phase modulation and vice versa, which is hard to keep track of and causes the new SLM to behave differently than intended. Moreover, the behavior of most multilevel SLMs changes strongly with the illumination wavelength, causing the proposed designs to operate in a satisfactory manner only for a narrow range of wavelengths.

To avoid such problems, one can consider the creation of full-complex SLMs out of binary SLMs [24]. Although the quantization constraint on binary SLMs is harsh, binary SLMs such as digital micromirror devices by Texas Instruments [25,26] provide the same on-off modulation regardless of the illumination wavelength, so they are more robust compared to multilevel SLMs, and their usage in multicolor applications, such as 3D displays, is easier. Moreover, binary SLMs seem to have a higher potential to be miniaturized compared to multilevel SLMs.

In this paper, we first propose a generic method for effectively creating full-complex SLMs out of binary SLMs, and then we propose a possible optical implementation for the generic method. We explain our generic method in Section 2. This method actually trades pixel count to dynamic range and 
carries out this trade-off by generalizing the concepts of bit plane representation and decomposition for ordinary digital gray scale multilevel images [27]. In particular, we propose to effectively obtain a $2^{K}$-level (or $K$ bit) SLM by forming a properly weighted superposition of $K$ binary (or 1 bit) SLMs. When $K$ is sufficiently large (such as $K=16$ ), the new SLM can be regarded as a full-complex SLM. We show that, in this way, information-wise, the binary SLMs are utilized in the most efficient manner possible. In Section 3, we propose a $4 f$ system to optically implement our generic method. With this system, out of a binary SLM with $P M \times Q N$ pixels, we can effectively create a $P Q$ bit (or $2^{P Q}$ level) SLM with $M \times N$ pixels. Again, when $P$ and $Q$ are sufficiently large (such as $P=Q=4$ ), the new SLM can be regarded as a full-complex one. We show that the $4 f$ system also provides a means for eliminating the disturbing higher orders from the diffraction field of the new SLM while preserving the central order with little distortion. The key element of the proposed $4 f$ system is an optical thin mask that needs to be physically produced and placed to the Fourier plane. In Section 4, we discuss several alternatives for this mask depending on the production capabilities and show that even very simple four-level, three-level, or binary masks work.

\section{GENERIC METHOD}

In this section, we explain our generic method for effectively creating full-complex SLMs out of binary SLMs. Here, we do not adhere to a specific optical system, but carry out the discussion at an abstract level. In Section 3 , we propose a possible optical implementation for our method. Our method is actually based on the well-known concepts of the bit plane representation and decomposition for ordinary digital gray scale images [27]. Therefore, we start with a quick review of these concepts.

Let $\mathbf{S}$ denote a $2^{K}$-level (or $K$ bit) gray scale digital image of size $M \times N\left(K, M, N \in \mathcal{Z}^{+}\right)$. Assume that the pixel values of $\mathbf{S}$ can be equal to $0,1,2, \ldots, 2^{K}-1$. Typically, taking $K=8$ is sufficient for high-quality images of daily life scenes. It is well known that $\mathbf{S}$ can be written as $\mathbf{S}=2^{0} \mathbf{B}_{\mathbf{0}}+$ $2{ }^{1} \mathbf{B}_{1}+\ldots+2{ }^{K-1} \mathbf{B}_{\mathbf{K}-\mathbf{1}}$, where $\mathbf{B}_{\mathbf{0}}, \mathbf{B}_{\mathbf{1}}, \ldots, \mathbf{B}_{\mathbf{K}-\mathbf{1}}$ are all $M \times N$ binary (or 1 bit) images each pixel of which can be equal to either 0 or 1 . These binary images are called the bit planes of $\mathbf{S}$, where $\mathbf{B}_{\mathbf{0}}$ is named the least significant bit plane and $\mathbf{B}_{\mathbf{K}-\mathbf{1}}$ is named the most significant bit plane. Given $\mathbf{S}$, writing it as $\mathbf{S}=2^{0} \mathbf{B}_{\mathbf{0}}+2{ }^{1} \mathbf{B}_{\mathbf{1}}+\ldots+2{ }^{K-1} \mathbf{B}_{\mathbf{K}-\mathbf{1}}$ is called the bit plane representation of $\mathbf{S}$ and the process of finding the appropriate $\mathbf{B}_{\mathbf{0}}, \mathbf{B}_{1}, \ldots, \mathbf{B}_{\mathbf{K}-1}$ is called the bit plane decomposition of $\mathbf{S}$. Note that in its bit plane representation, $\mathbf{S}$ is written as a particular weighted superposition of its bit planes, where the weights are given by $2^{0}, 2^{1}, \ldots, 2^{K-1}$. Our generic method for creating full-complex SLMs out of binary SLMs amounts to a generalization of this weighted superposition concept to include complex valued weighting coefficients as well.

Suppose we have $K$ binary SLMs of size $M \times N$ at hand. Assume the pixel periods, pixel geometries, and other physical parameters of all these SLMs are identical. Let us denote these binary SLMs with $\mathbf{B}_{\mathbf{0}}, \mathbf{B}_{\mathbf{1}}, \ldots, \mathbf{B}_{\mathbf{K}-\mathbf{1}}$. Let $\bar{b}_{i}[m, n]$ denote the value of the $(m, n)$ th pixel of $\mathbf{B}_{\mathbf{i}}$, where $0 \leq m \leq M-1$, $0 \leq n \leq N-1,0 \leq i \leq K-1$, and $m, n, i \in \mathcal{Z}$. Suppose $\bar{b}_{i}[m, n]$ can be set to either -1 or 1 . (Throughout this paper, we assume that binary SLM pixel values can be set to either
-1 or 1 . Extension of the presented results for other binary pixel values can be accomplished in a straightforward manner.) Now suppose, processing these binary SLMs with some kind of an optical system, we effectively form a new $M \times N$ SLM denoted by $\mathbf{S}$, such that $\mathbf{S}=w_{0} \mathbf{B}_{0}+$ $w_{1} \mathbf{B}_{1}+\ldots+w_{K-1} \mathbf{B}_{\mathbf{K}-1}$, where $w_{i} \in \mathcal{C}$ for $0 \leq i \leq K-1$, $i \in \mathcal{Z}$. Note that, if $\bar{s}[m, n]$ denotes the value of the $(m, n)$ th pixel of $\mathbf{S}$, we have $\bar{s}[m, n]=w_{0} \bar{b}_{0}[m, n]+$ $w_{1} \bar{b}_{1}[m, n]+\ldots+w_{K-1} \bar{b}_{K-1}[m, n]$. Hence, the new SLM is obtained as a weighted superposition of the binary SLMs where $w_{i}$ s denote the possibly complex valued weighting coefficients. Selecting these coefficients wisely, and taking $K$ as sufficiently large, we can make $\mathbf{S}$ a full-complex SLM.

As an example, suppose $K=16$, and suppose the weighting coefficients are taken as

$$
w_{i}= \begin{cases}\frac{1}{255} 2^{i} & \text { for } 0 \leq i \leq 7 \\ \frac{j}{255} 2^{i-8} & \text { for } 8 \leq i \leq 15\end{cases}
$$

with $j=\sqrt{-1}$. Then, adjusting $\bar{b}_{0}[m, n], \bar{b}_{1}[m, n], \ldots, \bar{b}_{15}[m, n]$, we can set each $\bar{s}[m, n]$ to any complex number of the form $\frac{1}{255}(R+j I)$, where $R, I=-255,-253,-251, \ldots, 251,253,255$. Note that the number of different complex values available for each $\bar{s}[m, n]$ is $2^{16}$ (corresponding to an information content of 16 bits per pixel, where with the current weights, 8 bits are reserved for the real part and 8 bits are reserved for the imaginary part). These complex values are displayed in Fig. 1(a). Since these complex numbers also have a good coverage of the complex plane, $\mathbf{S}$ can be regarded as a fullcomplex SLM. Hence, out of 16 binary (1 bit) SLMs of size $M \times N$, we have created a single full-complex (16 bit) SLM of size $M \times N$.

Continuing with the current example, note that, in the beginning, there are a total of $16 M N$ pixels. At the end, there are only $M N$ pixels, so the pixel count is reduced. However, the pixels in the beginning are binary (1 bit), while the pixels of the new SLM are full complex (16 bit), so the dynamic range is increased. Thus, we have essentially traded pixel count to dynamic range. Actually, information-wise, this trade-off is carried out in the most efficient manner possible. To see this, note that the information content of each of the binary SLMs is $M N$ bits, and since we use 16 binary SLMs, the total information content in the beginning is $16 M N$ bits. At the end, we obtain the full-complex SLM, which has $M N$ pixels, where each pixel has an information content of 16 bits, so the information content of the full-complex SLM is also $16 M N$ bits. Hence, the
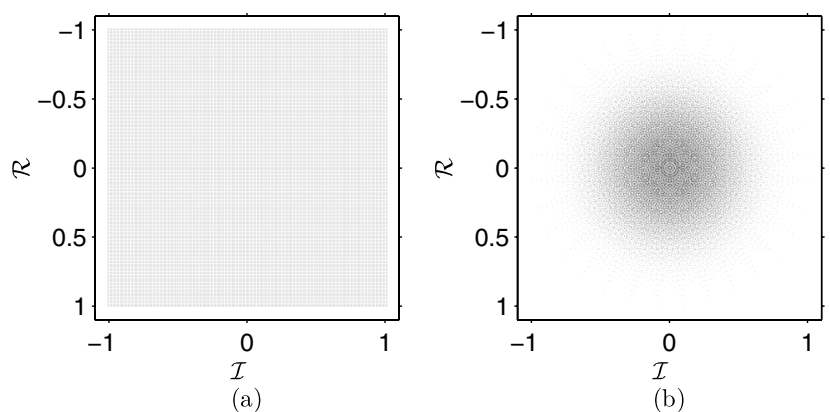

Fig. 1. (a) Complex numbers obtained with the weighting coefficients given in Eq. (1). (b) Complex numbers obtained with the weighting coefficients given in Eq. (2). 
full-complex SLM is created out of the binary SLMs without any loss in the information capacity. Therefore, informationwise, binary SLMs are utilized in the most efficient manner.

Actually, this nice result follows as a consequence of the fact that we create the binary SLM by forming a properly weighted superposition of the binary SLMs. That is, our choice of the weighting coefficients as in Eq. (1) is a wise choice. To see what happens when we do not choose these coefficients wisely, consider taking $w_{i}=1$ for all $i$, which corresponds to directly adding all 16 binary SLMs without using any weighting. If this were the case, then each pixel of $\mathbf{S}$ (obtained as $\mathbf{S}=\mathbf{B}_{\mathbf{0}}+\mathbf{B}_{1}+\ldots+\mathbf{B}_{15}$ ) could only be set to $-16,-14,-12, \ldots, 12,14,16$. So, only 17 different values would be available for each pixel of the new SLM, which is quite small compared to $2^{16}$ (which is the number of different values obtained with the former weights). Therefore, if we directly superposed the binary SLMs without any weighting, we would have used them in a quite inefficient manner while creating the new SLM (and it would not be possible to regard the new SLM as a full-complex one at all since only 17 different values would be available for each pixel). Therefore, a wise selection of the weighting coefficients is crucial in our method.

As we have seen, our initial selection for the weighting coefficients is a wise selection, but it is not the only possible wise selection. For instance, again suppose that $K=16$, and suppose the weighting coefficients are selected as

$$
w_{i}=\left|\frac{1-e^{\frac{j \pi}{16}}}{2}\right| e^{\frac{j i \pi}{16}}
$$

for $0 \leq i \leq 15, i \in \mathcal{Z}$. Using these coefficients, each pixel of $\mathbf{S}$ can be set to one of the complex numbers displayed in Fig. 1(b). These complex numbers are different from the ones shown in Fig. 1(a), but again there are $2^{16}$ different complex numbers on this figure and they have a good coverage of the complex plane. Thus, the new coefficients can also be used to create a 16 bit full-complex SLM as well. Many other nice selections (not listed here) for the weighting coefficients are also possible.

Note that our choice of $K$ and $w_{i}$ s determines the values that are available for a pixel of $\mathbf{S}$. That is, if during some application, the desired value for a pixel of $\mathbf{S}$ is not among the available values, we should first perform a quantization. Therefore, our choice of $K$ and $w_{i}$ s actually defines a quantizer on the complex plane. In order to achieve optimum performance, we should design this quantizer (i.e., choose $K$ and $w_{i}$ s) by taking into account the statistical properties of the analog source. Earlier, we provided examples for the $K=16$ case. However, in some applications, smaller values of $K$ can be sufficient. For instance, in [4,28], it is discussed that even 4 bit quantization can be quite sufficient for holographic applications.

In summary, our generic method for creating full-complex SLMs out of binary SLMs can be described as follows: Using some optical system, effectively form a weighted superposition of $K$ binary SLMs to obtain a new SLM, where $K$ is sufficiently large. Select the weights such that each pixel of the new SLM can be set to $2^{K}$ different complex values, where these values also have a satisfactory coverage of the complex plane. Under these conditions, the new SLM can be regarded as a full-complex one. In this way, information-wise, the binary SLMs are utilized in the most efficient manner while creating the full-complex SLM.

Before closing this section, let us discuss how to configure the binary SLMs in order to make the new SLM $\mathbf{S}$ equal to some desired full-complex SLM denoted by $\mathbf{S}_{\mathbf{d}}$. That is, we wish to determine $\mathbf{B}_{\mathbf{0}}, \mathbf{B}_{\mathbf{1}}, \ldots, \mathbf{B}_{\mathbf{K}-\mathbf{1}}$ such that we achieve $\mathbf{S}=\mathbf{S}_{\mathbf{d}}$, where $\mathbf{S}=w_{0} \mathbf{B}_{\mathbf{0}}+w_{1} \mathbf{B}_{\mathbf{1}}+\ldots+w_{K-1} \mathbf{B}_{\mathbf{K}-\mathbf{1}}$. Let us assume that the weighting coefficients are selected wisely such as in Eq. (1) or in Eq. (2), so that each pixel of $\mathbf{S}$ can be set to $2^{K}$ different complex values. Let us also assume that $\mathbf{S}_{\mathbf{d}}$ is already quantized. Then, for each pixel of $\mathbf{S}_{\mathbf{d}}$, we should solve the equation $\bar{s}_{d}[m, n]=\sum_{i=0}^{K-1} w_{i} \bar{b}_{i}[m, n]$ and determine $\bar{b}_{i}[m, n]$ under the constraint that $\bar{b}_{i}[m, n]= \pm 1$. An easy method is to prepare a lookup table that holds the mapping between the possible binary patterns of size $1 \times K$ and the complex numbers produced by them and use this lookup table to determine $\bar{b}_{0}[m, n], \bar{b}_{1}[m, n], \ldots, \bar{b}_{K-1}[m, n]$. Note that this lookup table will have $2^{K}$ entries. When $K \leq 16$, such a lookup table can be handled easily with today's computation and storing technology. If the achievable complex numbers are listed in the lookup table in an intelligent manner, search times may be minimized or, in certain cases, no search may be needed at all.

\section{PRACTICAL IMPLEMENTATION USING A $4 f$ SYSTEM}

In the previous section, we described our generic method for creating a $K$ bit SLM out of $K$ binary SLMs. We carried that discussion at an abstract level without adhering to a specific optical system and concluded that we can successfully create the full-complex SLM if we have some kind of an optical system that effectively forms a properly weighted superposition of $K$ binary SLMs. In this section, we propose an optical system that forms this weighted superposition. We assume that we have a binary SLM of size $P M \times Q N$ at hand, and out of it, we will create a $P Q$ bit SLM of size $M \times N$, where $M, N, P, Q \in \mathcal{Z}^{+}$. While doing so, we will view the binary SLM as a collection of $P Q$ sub-SLMs, where each sub-SLM is also binary and has size $M \times N$. We will form the weighted superposition of these sub-SLMs to obtain the $P Q$ bit SLM. We explain our optical system in three subsections. In Subsection 3.A, we propose a simple linear shift invariant (LSI) system through which we obtain the weighted superposition of the sub-SLMs. In Subsection 3.B, we consider a bandlimited version of the LSI system we propose in Subsection 3.A because although we planned a $4 f$ system-based implementation, the initial LSI system has infinite bandwidth, so it is not possible to practically implement. In Subsection 3.C, we propose a $4 f$ system that implements the bandlimited $\overline{\mathrm{LSI}}$ system considered in Subsection 3.B.

\section{A. LSI System to Form the Weighted Superposition of Binary SLMs}

Consider a binary SLM that has $P M \times Q N$ pixels. Let $\Delta_{x}$ and $\Delta_{y}$ denote the pixel periods of the SLM. Let $a(x, y)$ denote the pixel aperture function of the SLM such that $a(x, y)=0$ for $x \notin\left[0, \Delta_{x}\right]$ or $y \notin\left[0, \Delta_{y}\right]$. In most cases, $a(x, y)=\operatorname{rect}\left(\frac{x}{\Delta_{x}}-0.5\right) \operatorname{rect}\left(\frac{y}{\Delta_{y}}-0.5\right)$, where $\operatorname{rect}(x)=1$ for $|x|<0.5, \operatorname{rect}(x)=0.5$ for $|x|=0.5$, and $\operatorname{rect}(x)=0$ for $|x|>0.5$. Let $\bar{b}[m, n]$ denote the value of the $(m, n)$ th SLM pixel $(m, n \in \mathcal{Z})$ for $0 \leq m \leq P M-1$ and $0 \leq n \leq Q N-1$ such 
that $\bar{b}[m, n]$ can be set to either -1 or 1 . If $b(x, y)$ denotes the complex transmittance of the binary SLM, we have

$$
b(x, y)=a(x, y) * * \sum_{m=0}^{P M-1} \sum_{n=0}^{Q N-1} \bar{b}[m, n] \delta\left(x-m \Delta_{x}, y-n \Delta_{y}\right)
$$

In Eq. (3), ** denotes the $2 \mathrm{D}$ convolution operation such that $f_{1}(x, y) * * f_{2}(x, y)=\int_{-\infty}^{\infty} \int_{-\infty}^{\infty} f_{1}\left(x^{\prime}, y^{\prime}\right) f_{2}\left(x-x^{\prime}\right.$, $\left.y-y^{\prime}\right) \mathrm{d} x^{\prime} \mathrm{d} y^{\prime}$. With the definitions above, the SLM is assumed to lie in the region $0 \leq x \leq P M \Delta_{x}$ and $0 \leq y \leq Q N \Delta_{y}$. Note that we can view our binary SLM as a collection of $P Q$ sub-SLMs (which are also binary) where each sub-SLM consists of $M \times N$ pixels. In this respect, we can write $b(x, y)$ as

$$
b(x, y)=\sum_{p=0}^{P-1} \sum_{q=0}^{Q-1} b_{p, q}\left(x-p M \Delta_{x}, y-q N \Delta_{y}\right)
$$

where $b_{p, q}(x, y)$ denotes the complex transmittance of the $(p, q)$ th sub-SLM and is given as

$$
\begin{aligned}
b_{p, q}(x, y)= & b\left(x+p M \Delta_{x}, y+q N \Delta_{y}\right) \operatorname{rect}\left(\frac{x-0.5 M \Delta_{x}}{M \Delta_{x}}\right) \\
& \times \operatorname{rect}\left(\frac{y-0.5 N \Delta_{y}}{N \Delta_{y}}\right)
\end{aligned}
$$

for $0 \leq p \leq P-1$ and $0 \leq q \leq Q-1$. Note that all sub-SLMs have pixel aperture function $a(x, y)$ and pixel periods $\Delta_{x}$ and $\Delta_{y}$.
When forming the binary SLM, the $(p, q)$ th sub-SLM is placed in the region $p M \Delta_{x} \leq x \leq(p+1) M \Delta_{x}$ and $q N \Delta_{y} \leq y \leq$ $(q+1) N \Delta_{y}$ [a one-dimensional (1D) illustration is provided in Fig. 2, where $P=4$ and the sub-SLMs are denoted by $b_{0}$, $b_{1}, b_{2}$, and $b_{3}$. Our purpose is to form a weighted superposition of these sub-SLMs. We will accomplish this by processing $b(x, y)$ with a suitably defined LSI system.

Consider an LSI system whose impulse response $h(x, y)$ is given as

$$
h(x, y)=\sum_{p=0}^{P-1} \sum_{q=0}^{Q-1} \bar{w}[p, q] \delta\left(x-p M \Delta_{x}, y-q N \Delta_{y}\right)
$$

where $\bar{w}[p, q] \in \mathcal{C}$ for $0 \leq p \leq P-1,0 \leq q \leq Q-1, p, q \in \mathcal{Z}$. As seen, $h(x, y)$ consists of a $P \times Q$ grid of impulses that are spaced by $M \Delta_{x}$ and $N \Delta_{y}$. The $(p, q)$ th impulse is located at $\left(p M \Delta_{x}, q N \Delta_{y}\right)$ and has strength $\bar{w}[p, q]$. If $H\left(\nu_{x}, \nu_{y}\right)$ denotes the frequency response of this LSI system, we have

$$
\begin{aligned}
H\left(\nu_{x}, \nu_{y}\right) & =\mathcal{F}\{h(x, y)\} \\
& =\int_{-\infty}^{\infty} \int_{-\infty}^{\infty} h(x, y) \exp \left\{-j 2 \pi\left(x \nu_{x}+y \nu_{y}\right)\right\} \mathrm{d} x \mathrm{~d} y \\
& =\sum_{p=0}^{P-1} \sum_{q=0}^{Q-1} \bar{w}[p, q] e^{-j 2 \pi\left\{\nu_{x} p M \Delta_{x}+\nu_{y} q N \Delta_{y}\right\}} .
\end{aligned}
$$

It is easy to see that $H\left(\nu_{x}, \nu_{y}\right)$ is periodic with periods $\frac{1}{M \Delta_{x}}$ and $\frac{1}{N \Delta_{y}}$.

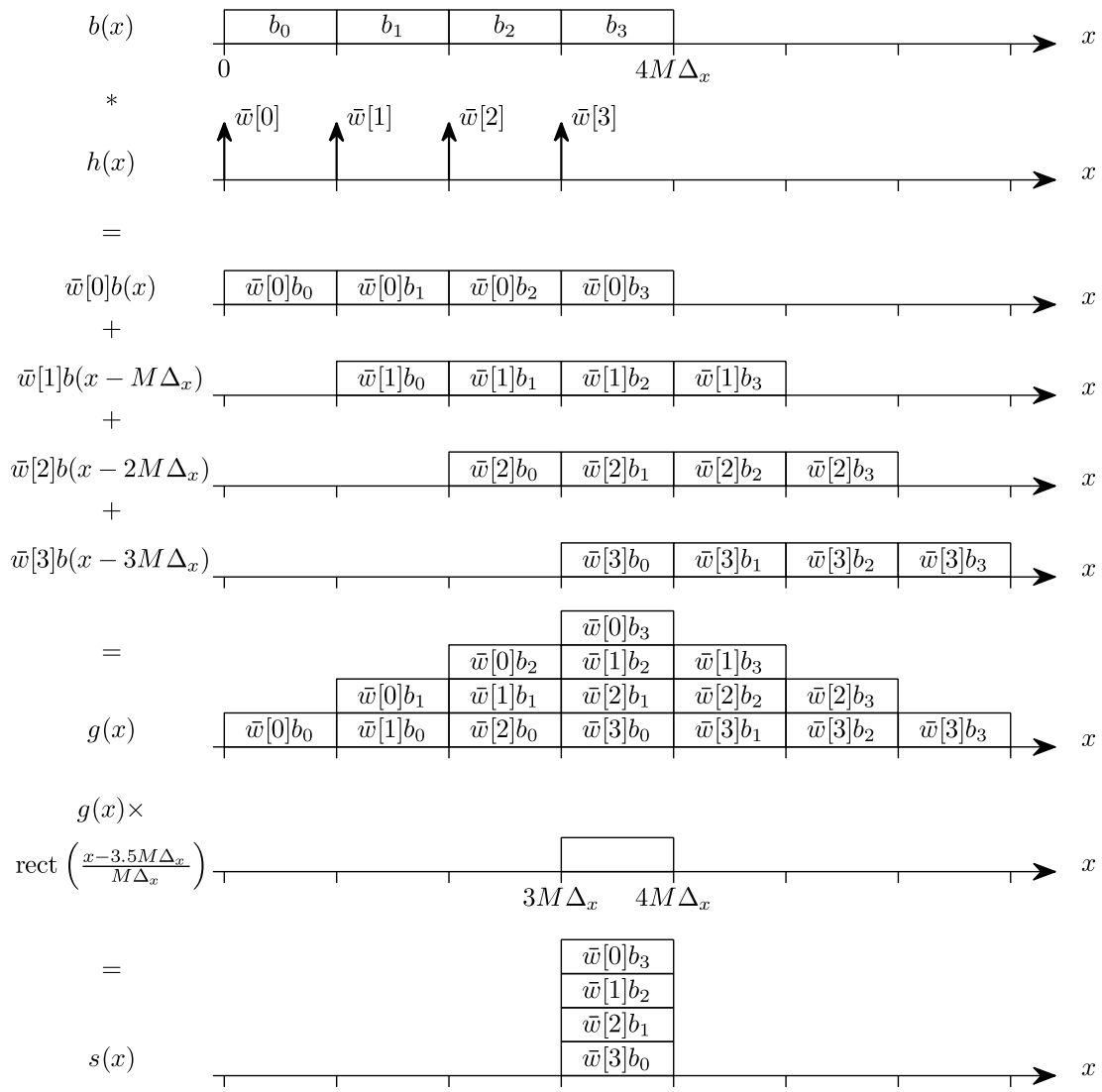

Fig. 2. 1D illustration of the process through which $s(x, y)$ is created out of $b(x, y) . b(x), h(x), g(x), s(x)$, and $\bar{w}[p]$ respectively denote the 1D counterparts of $b(x, y), h(x, y), g(x, y), s(x, y)$, and $\bar{w}[p, q]$, which are discussed in the text. In the 1D case, we assumed that the binary SLM is divided into four sub-SLMs of size $M$, and denoted these sub-SLMs with $b_{0}, b_{1}, b_{2}$, and $b_{3}$. 
Suppose $b(x, y)$ is processed by this LSI system. Let $g(x, y)$ denote the resulting output such that $g(x, y)=$ $b(x, y) * * h(x, y)$. Then we can write

$$
g(x, y)=\sum_{p=0}^{P-1} \sum_{q=0}^{Q-1} \bar{w}[p, q] b\left(x-p M \Delta_{x}, y-q N \Delta_{y}\right) .
$$

The spatial support of $g(x, y)$ is given by the region $0 \leq x \leq$ $(2 P-1) M \Delta_{x}$ and $0 \leq y \leq(2 Q-1) N \Delta_{y}$. Examining $g(x, y)$, we see that the LSI system actually forms a superposition of shifted and weighted replicas of $b(x, y)$. Such LSI systems are usually called echo systems in the signal processing literature, since $1 \mathrm{D}$ versions of them are used to produce synthetically generated echoes of sound signals in audio processing.

Now, define $s(x, y)$ such that

$$
\begin{aligned}
s(x, y)= & g(x, y) \operatorname{rect}\left(\frac{x-(P-0.5) M \Delta_{x}}{M \Delta_{x}}\right) \\
& \times \operatorname{rect}\left(\frac{y-(Q-0.5) N \Delta_{y}}{N \Delta_{y}}\right) .
\end{aligned}
$$

Hence, $s(x, y)$ is obtained by windowing $g(x, y)$ in space. The window selects the portion of $g(x, y)$ lying in the region $(P-1) M \Delta_{x} \leq x \leq P M \Delta_{x}$ and $(Q-1) N \Delta_{y} \leq y \leq Q N \Delta_{y}$. It is straightforward to show that

$$
s(x, y)=\sum_{p=0}^{P-1} \sum_{q=0}^{Q-1} \bar{w}[P-1-p, Q-1-q] b_{p, q}\left(x-x_{0}, y-y_{0}\right)
$$

with $x_{0}=(P-1) M \Delta_{x}$ and $y_{0}=(Q-1) N \Delta_{y}$.

Equation (10) is the result we have been seeking. We see that $s(x, y)$ is obtained as the weighted superposition of $b_{p, q}(x, y)$, where the weights are given by $\bar{w}[p, q]$ (a 1D illustration of the process through which $s(x, y)$ is obtained from $b(x, y)$ is provided in Fig. 2 for $P=4)$. Hence, $s(x, y)$ represents the complex transmittance of a new SLM that is obtained as the weighted superposition of the sub-SLMs of the binary SLM. Note that the new SLM also has the pixel aperture function $a(x, y)$ and pixel periods $\Delta_{x}$ and $\Delta_{y}$. It lies in the region $(P-1) M \Delta_{x} \leq x \leq P M \Delta_{x}$ and $(Q-1) N \Delta_{y} \leq y \leq Q N \Delta_{y}$. We know from Section $\underline{2}$ that, properly choosing $\bar{w}[p, q]$, we can make this new SLM a $P Q$ bit SLM. We also know that when $P$ and $Q$ are chosen sufficiently large (such as $P=$ $Q=4$ ), the new SLM can be regarded as a full-complex one. Hence, using the proposed LSI system, we can create an $M \times$ $N$ full-complex ( $P Q$ bit) SLM out of a $P M \times Q N$ binary SLM.

As an illustration for the $P=Q=4$ and $M=N=256$ case, Fig. 3 shows a $1024 \times 1024$ binary SLM. Figures $\underline{4}$ and 5 respectively show the real and imaginary parts of $g(x, y)=\bar{h}(x, y) *$ $* b(x, y)$ when $\bar{w}[p, q]$ are taken as

$$
\begin{gathered}
{\left[\begin{array}{cccc}
\bar{w}[0,0] & \bar{w}[0,1] & \bar{w}[0,2] & \bar{w}[0,3] \\
\bar{w}[1,0] & \bar{w}[1,1] & \bar{w}[1,2] & \bar{w}[1,3] \\
\bar{w}[2,0] & \bar{w}[2,1] & \bar{w}[2,2] & \bar{w}[2,3] \\
\bar{w}[3,0] & \bar{w}[3,1] & \bar{w}[3,2] & \bar{w}[3,3]
\end{array}\right]} \\
=\frac{1}{255}\left[\begin{array}{cccc}
j 2^{7} & j 2^{6} & j 2^{5} & j 2^{4} \\
j 2^{3} & j 2^{2} & j 2^{1} & j 2^{0} \\
2^{7} & 2^{6} & 2^{5} & 2^{4} \\
2^{3} & 2^{2} & 2^{1} & 2^{0}
\end{array}\right] .
\end{gathered}
$$

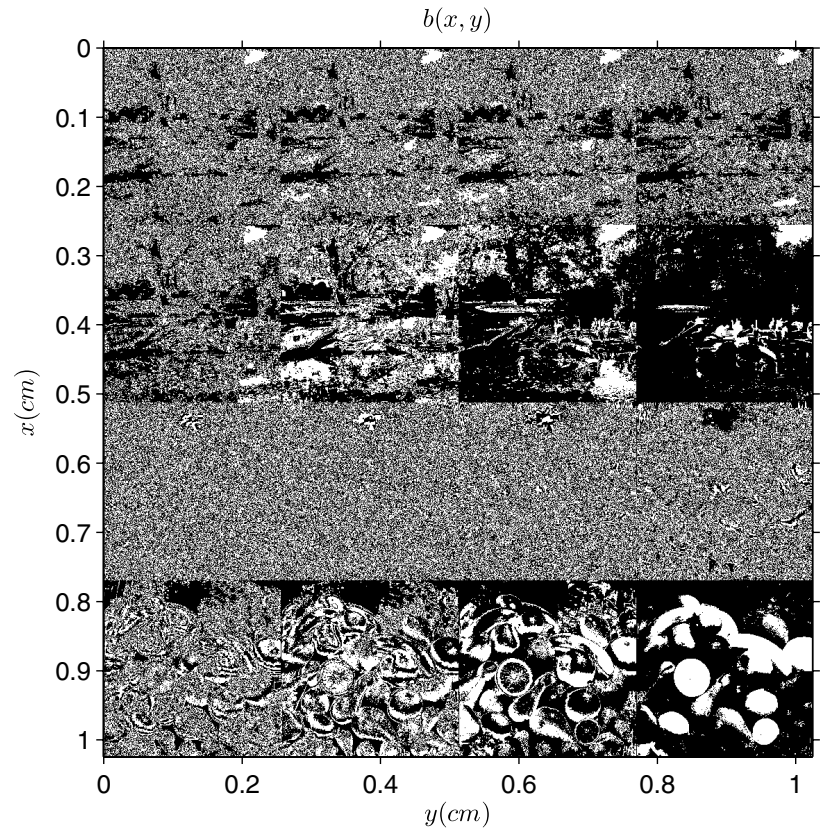

Fig. 3. Binary SLM pattern.

Figures 6 (a) and $6(\mathrm{~b})$, which are respectively the magnified versions of the signals within the windows in Figs. $\underline{4}$ and $\underline{5}$, show the real and imaginary parts of $s(x, y)$. As seen, a $256 \times 256$ full-complex SLM has been successfully created out of the binary SLM. (In this example, we took $\Delta_{x}=\Delta_{y}=$ $10 \mu \mathrm{m}$ and assumed rectangular pixels. In Fig. $\underline{3}$, black pixels have value -1 and white pixels have value 1 .)

\section{B. Imposing a Bandwidth Limitation}

In theory, the LSI system proposed in Subsection 3.A enables us to effectively create a $P Q$ bit $M \times N$ SLM out of a $P M \times Q N$ binary SLM. It is well known that $4 f$ systems can be used to optically implement LSI systems, and we do so in Subsection 3.C. However, before proceeding, we analyze

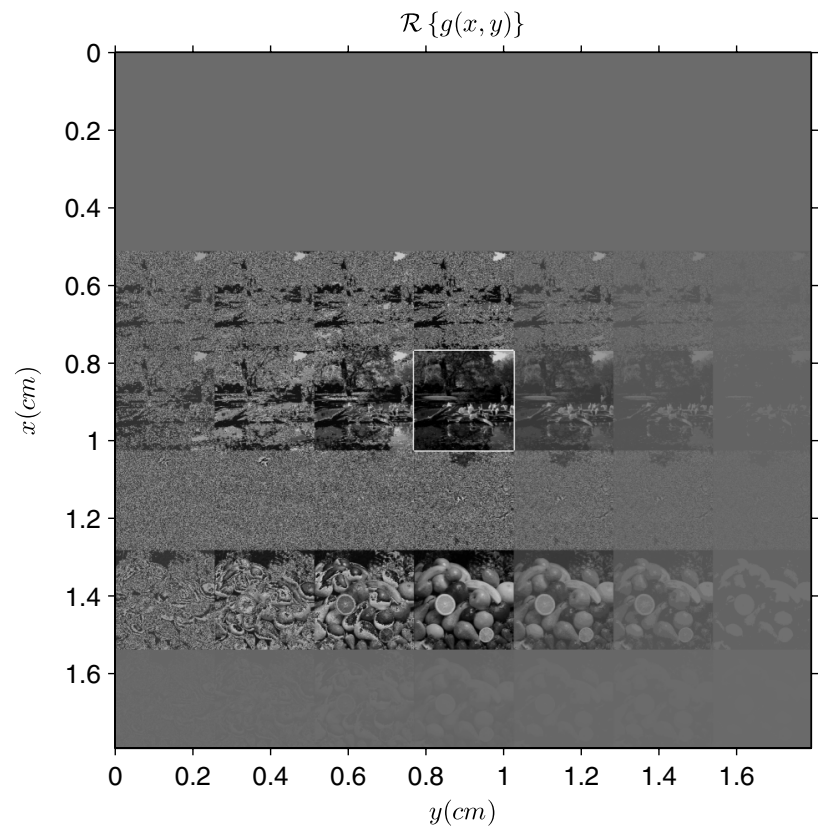

Fig. 4. Real part of $g(x, y)$. 


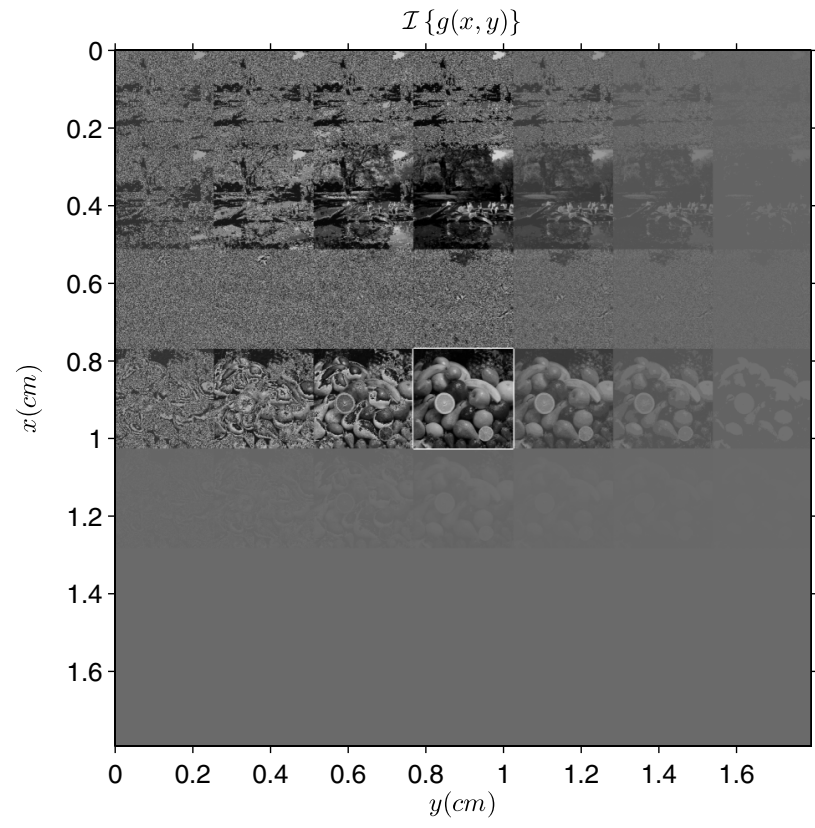

Fig. 5. Imaginary part of $g(x, y)$.

the effects of imposing a bandwidth restriction to the LSI system we used in Subsection 3.A. The reason is, the frequency response $H\left(\nu_{x}, \nu_{y}\right)$ of that $\overline{\mathrm{LSI}}$ system occupies the entire frequency spectrum, whereas a $4 f$ setup that consists of finite-sized lenses and optical masks can only support a finite bandwidth. In this respect, let us consider a new LSI system with impulse response $h_{L}(x, y)$ such that

$$
h_{L}(x, y)=h(x, y) * * h_{B}(x, y),
$$

where $h_{B}(x, y)$ denotes the impulse response of an ideal lowpass filter with bandwidths $B_{x}$ and $B_{y}$ such that

$$
h_{B}(x, y)=B_{x} B_{y} \operatorname{sinc}\left(x B_{x}\right) \operatorname{sinc}\left(y B_{y}\right)
$$

with $\operatorname{sinc}(x)=\frac{\sin (\pi x)}{\pi x}$. Hence, the new LSI system is the bandlimited version of the original LSI system. Note that, if $H_{L}\left(\nu_{x}, \nu_{y}\right)$ and $H_{B}\left(\nu_{x}, \nu_{y}\right)$ respectively denote the Fourier transforms of $h_{L}(x, y)$ and $h_{B}(x, y)$, we have $H_{B}\left(\nu_{x}, \nu_{y}\right)=$ $\operatorname{rect}\left(\frac{\nu_{x}}{B_{x}}\right) \operatorname{rect}\left(\frac{\nu_{y}}{B_{y}}\right)$ and $H_{L}\left(\nu_{x}, \nu_{y}\right)=H\left(\nu_{x}, \nu_{y}\right) H_{B}\left(\nu_{x}, \nu_{y}\right)$. Let $g_{L}(x, y)$ denote the output when the binary SLM is processed by the new LSI system, so that $g_{L}(x, y)=b(x, y) * * h_{L}(x, y)$. We can also write

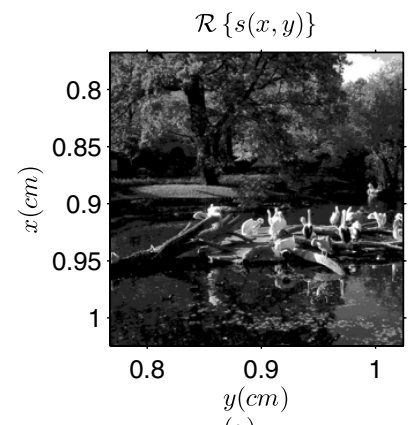

(a)

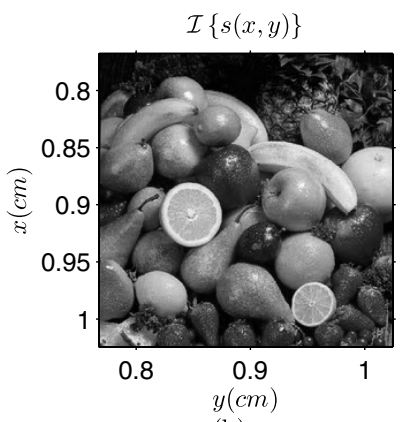

(b)
Fig. 6. (a) Real and (b) imaginary parts of $s(x, y)$.

$$
g_{L}(x, y)=g(x, y) * * h_{B}(x, y),
$$

which indicates that the output of the new LSI system is a blurred version of the output of the original LSI system. Recall that when the original LSI system was used, the full-complex SLM [represented by $s(x, y)$ ] was selected out of $g(x, y)$ with a simple windowing operation in space. Suppose we apply the same window to $g_{L}(x, y)$ and denote the resulting output with $s_{L}(x, y)$ such that $s_{L}(x, y)=$ $g_{L}(x, y) \operatorname{rect}\left(\frac{x-(P-0.5) M \Delta_{x}}{M \Delta_{x}}\right) \operatorname{rect}\left(\frac{y-(Q-0.5) N \Delta_{y}}{N \Delta_{y}}\right)$. Assuming that the blurring is not too strong (i.e., $B_{x}$ and $B_{y}$ are sufficiently large, or $h_{B}(x, y)$ is sufficiently narrow) so that leakages due to infinite tails of $h_{B}(x, y)$ can be ignored, we can write

$$
s_{L}(x, y) \approx s(x, y) * * h_{B}(x, y)
$$

Hence, when the new LSI system is used, we approximately obtain a blurred version of the full-complex SLM pattern represented by $s(x, y)$. Since the free space propagation is also a LSI system, the light field produced by the SLM at any distance will also experience the same blurring. Obviously, we do not want to lose any important information present in the generated light field due to this blurring effect. Thus, there is a limit to the degree of blurring we can tolerate. It is well known that the light field produced by a pixellated SLM consists of diffraction orders, which are shifted, modulated, and dispersed versions of each other, so they essentially carry the same information $[15,29,30]$. The order that has the lowest frequency content is called the central order. The blurring will not cause any information loss as long as the central order remains unaffected from it. This is the case if $B_{x}$ and $B_{y}$ are greater than the bandwidths of the central order, which are given as $\frac{1}{\Delta_{x}}$ and $\frac{1}{\Delta_{y}}$ in our case. Hence, the bandlimited LSI system can be confidently used instead of the original LSI system if

$$
B_{x}>\frac{1}{\Delta_{x}} \quad \text { and } \quad B_{y}>\frac{1}{\Delta_{y}} \text {. }
$$

Indeed, if the above conditions are met near the limit (i.e., $B_{x} \approx \frac{1}{\Delta_{x}}$ and $B_{y} \approx \frac{1}{\Delta_{y}}$ ), central order of the light field produced by the full-complex SLM is preserved (with little distortion) while higher orders are almost eliminated. This result may be explicitly desired in certain applications, such as $3 \mathrm{D}$ displays, where presence of higher orders is disturbing. As for an illustration, assume $P=Q=4, M=N=256$, and consider the $1024 \times 1024$ binary SLM depicted in Fig. 7 ( $\Delta_{x}=\Delta_{y}=10 \mu \mathrm{m}$, the SLM has rectangular pixels). Suppose the weighting coefficients are as given in Eq. (11). If we processed this binary SLM with the original LSI system (no band limitation), we would obtain the full-complex SLM depicted in Fig. 8(a), which would produce the diffraction field depicted in Fig. 8 (b) at a distance of $50 \mathrm{~cm}$. Note that, since the SLM in Fig. 8(a) has rectangular pixels, the diffraction field in Fig. $8(\mathrm{~b})$ consists of diffraction orders (the bright guitar at the center is the central diffraction order while its replicas are the higher diffraction orders). Next, Fig. 8(c) depicts the blurred version of the full-complex SLM depicted in Fig. 8(a) that we obtain when we process the binary SLM with the new LSI system with bandwidths given by $B_{x}=\frac{1}{\Delta_{x}}$ and $B_{y}=\frac{1}{\Delta_{y}}$. Figure $8(\mathrm{~d})$ displays the new diffraction field. As explained earlier, the central order is almost unaffected from the 


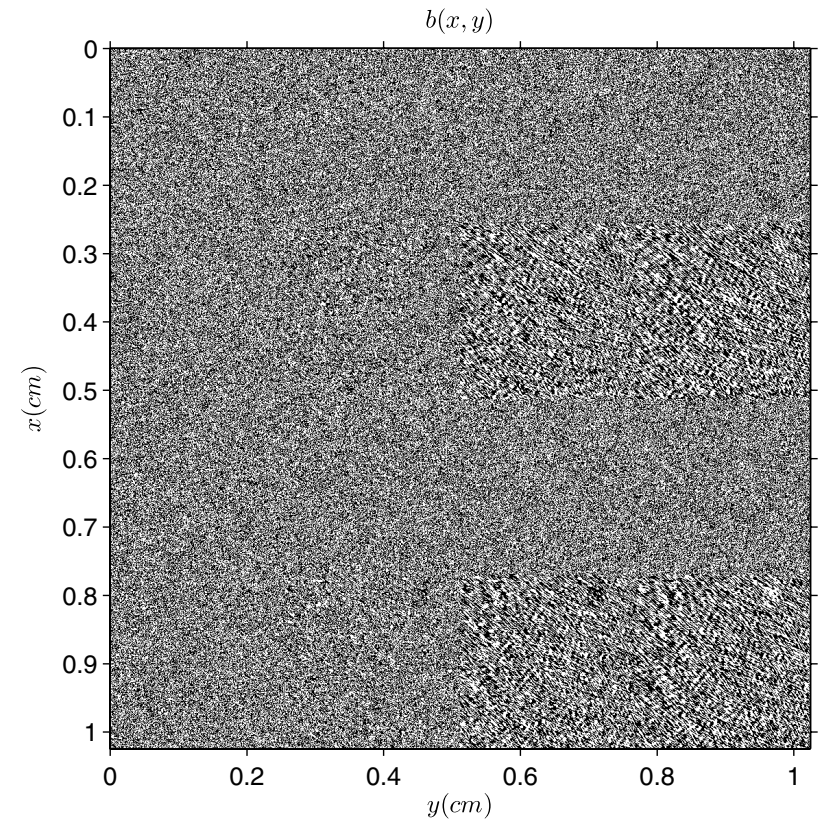

Fig. 7. Binary SLM pattern.

blurring, while the higher orders are almost eliminated. [In these figures, $h_{z}(x, y)$ denotes the impulse response of free space propagation. We computed the diffraction fields taking $h_{z}(x, y)=(j \lambda z)^{-1} e^{\frac{j 2 \pi z}{\lambda}} \exp \left\{\frac{j \pi}{\lambda z}\left(x^{2}+y^{2}\right)\right\}$, which is the impulse response of the commonly used Fresnel approximation with $z$ denoting the propagation distance and $\lambda$ denoting the wavelength. We took $\lambda=632.9 \mathrm{~nm}$.]

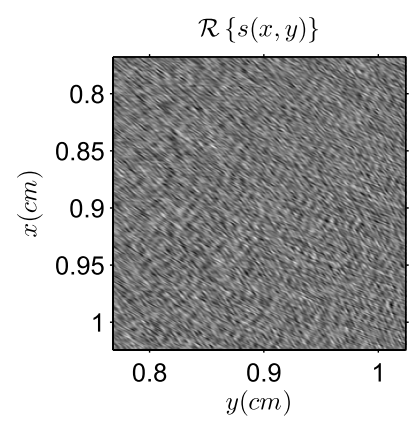

(a)

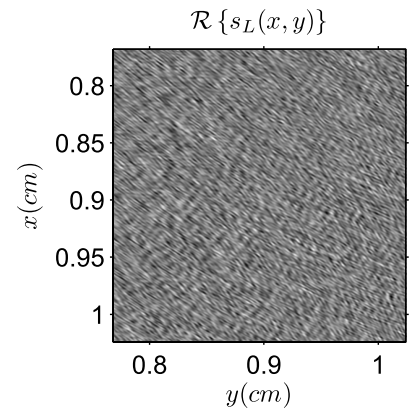

(c)

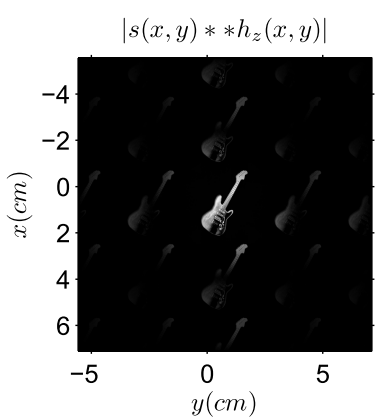

(b)

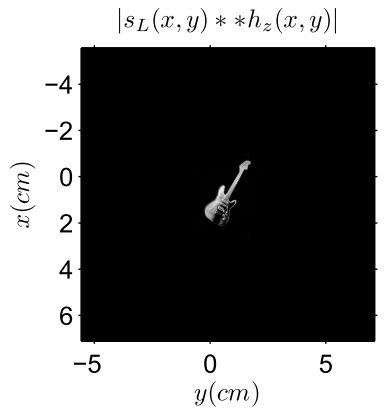

(d)
Fig. 8. (a) Full-complex SLM pattern obtained by processing the binary SLM pattern in Fig. 7 with the LSI system described by $h(x, y)$. (b) Resulting diffraction field at $50 \mathrm{~cm}$. (c) Full-complex SLM pattern obtained with the LSI system described by $h_{B}(x, y)$. (d) Resulting diffraction field at $50 \mathrm{~cm}$.

\section{Implementation with a $4 \boldsymbol{f}$ System}

Now, the ground for optical implementation is established. For a single wavelength, the LSI system described by Eq. (12) can be optically implemented using a $4 f$ system. Consider the system depicted in Fig. 9. As seen, two positive thin lenses (denoted by $L_{1}$ and $\left.L_{2}\right)$ with focal lengths $f(f>0)$ are placed at $z=f$ and $z=3 f$ planes. If the illumination wavelength is $\lambda$, the complex transmittances of these lenses are given by

$$
t_{l}(x, y)=\exp \left\{-\frac{j \pi}{\lambda f}\left(x^{2}+y^{2}\right)\right\}
$$

At the Fourier plane ( $z=2 f$ plane), an optical thin mask (that we name the Fourier plane mask) denoted by $M_{F}$ is placed. Let $m_{F}(x, y)$ denote the complex transmittance of this mask. At the output plane ( $z=4 f$ plane), another thin mask (that we name the output plane mask) denoted by $M_{O}$ is placed. Let $m_{O}(x, y)$ denote the complex transmittance of this mask. We assume that both of these masks are passive components, implying that their magnitude transmission at any point should be less than or equal to unity. We simply assume that $\max \left\{\left|m_{F}(x, y)\right|\right\}=1$ and $\max \left\{\left|m_{O}(x, y)\right|\right\}=1$. Let $u_{0}(x, y)$ denote the light field over the input plane $(z=0$ plane). As explained in [31], according to the Fresnel scalar diffraction theory, the light field just to the left of the output plane mask is given as

$$
u_{4 f^{-}}(x, y)=\frac{e^{\frac{j \delta \pi f}{\lambda}}}{(j \lambda f)^{2}} u_{0}(-x,-y) * * M_{F}\left(\frac{x}{\lambda f}, \frac{y}{\lambda f}\right),
$$

where $M_{F}\left(\nu_{x}, \nu_{y}\right)$ denotes the Fourier transform of $m_{F}(x, y)$. We see that if we take $u_{0}(x, y)=j^{2} e^{\frac{-j 8 \pi f}{\lambda}} b(-x,-y)$ (which corresponds to placing the $180^{\circ}$ rotated version of the binary SLM pattern to the third quadrant of the input plane and illuminating it with a normally incident plane wave of complex amplitude $j^{2} e^{\frac{-j 8 \pi f}{\lambda}}$ ) and if we have $M_{F}\left(\frac{x}{\lambda f}, \frac{y}{\lambda f}\right)=\frac{1}{\eta}(\lambda f)^{2} h_{L}(x, y)$ (where $\frac{1}{\eta}$ is included to satisfy the passive mask condition), we can obtain $u_{4 f^{-}}(x, y)=\frac{1}{\eta} g_{L}(x, y)$. Therefore, we should have

$$
\begin{aligned}
m_{F}(x, y)= & \frac{1}{\eta} H_{L}\left(-\frac{x}{\lambda f},-\frac{y}{\lambda f}\right) \\
= & \frac{1}{\eta} H_{B}\left(-\frac{x}{\lambda f},-\frac{y}{\lambda f}\right) H\left(-\frac{x}{\lambda f},-\frac{y}{\lambda f}\right) \\
= & \frac{1}{\eta} \operatorname{rect}\left(\frac{x}{W_{x}}\right) \\
& \times \operatorname{rect}\left(\frac{y}{W_{y}}\right) \sum_{p=0}^{P-1} \sum_{q=0}^{Q-1} \bar{w}[p, q] e^{\frac{j 2 \pi}{\lambda f}\left\{x p M \Delta_{x}+y q N \Delta_{y}\right\}},
\end{aligned}
$$

where $W_{x}=B_{x} \lambda f, W_{y}=B_{y} \lambda f$. It is easy to see that $m_{F}(x, y)$ corresponds to the complex transmittance of a periodic grating that is windowed in space, where the grating periods are $\frac{\lambda f}{M \Delta_{x}}$ and $\frac{\lambda f}{N \Delta_{y}}$ and the window widths are $W_{x}$ and $W_{y}$. Because of Eq. (16), we should have $W_{x}>\frac{\lambda f}{\Delta_{x}}$ and $W_{y}>\frac{\lambda f}{\Delta_{y}}$, so at least $M \times N$ periods of the grating should be preserved after windowing. For the weights given in Eq. $(\underline{11}), m_{F}(x, y)$ is illustrated in Fig. 10(a) for five periods in each dimension. (In this figure, $P \overline{=Q}=4, M=N=256, \Delta_{x}=\Delta_{y}=10 \mu \mathrm{m}$, 


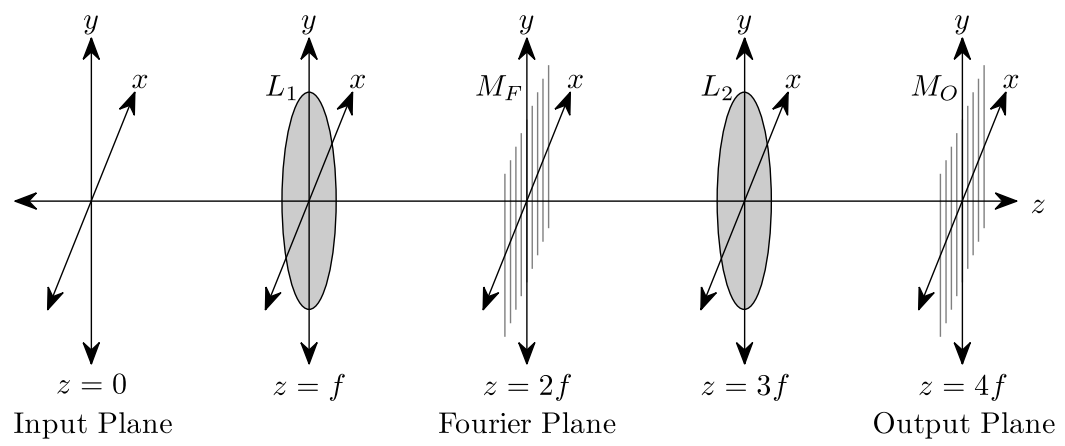

Fig. 9. $4 f$ setup. $L_{1}$ and $L_{2}$ denote positive thin lenses of focal length $f . M_{F}$ and $M_{O}$ respectively denote the Fourier and output plane masks.

$f=10 \mathrm{~cm}, \lambda=632.9 \mathrm{~nm}$.) Recall that the $\frac{1}{\eta}$ factor is included in Eq. (19) to ensure that $\max \left\{\left|m_{F}(x, y)\right|\right\}=1$. The value of $\eta$ is given as

$$
\eta=\max \left\{\left|\sum_{p=0}^{P-1} \sum_{q=0}^{Q-1} \bar{w}[p, q] e^{j 2 \pi\left\{x^{\prime} p+y^{\prime} q\right\}}\right|\right\}
$$

for $x^{\prime}, y^{\prime} \in \mathcal{R}$.

Finally, to select $\frac{1}{\eta} s_{L}(x, y)$ out of $u_{4 f^{-}}(x, y)=\frac{1}{\eta} g_{L}(x, y)$, we can use the following simple output plane mask

$m_{O}(x, y)=\operatorname{rect}\left(\frac{x-(P-0.5) M \Delta_{x}}{M \Delta_{x}}\right) \operatorname{rect}\left(\frac{y-(Q-0.5) N \Delta_{y}}{N \Delta_{y}}\right)$.

At the end, we obtain $u_{4 f^{+}}(x, y)=u_{4 f^{-}}(x, y) m_{O}(x, y) \approx$ $\frac{1}{\eta} s_{L}(x, y)$ as desired.

\section{Discussion about the $4 f$ Setup}

First, we should remind that the proposed $4 f$ setup is analyzed using Fresnel scalar diffraction theory, which is accurate under paraxial cases, i.e., the light rays traveling throughout the system must be confined to the vicinity of the optical axis and they should have small angles. Hence, the physical optical setup must be prepared accordingly. The binary SLM size should not be too large, and the focal length of the positive lenses should not be too small. Usually, these are already a straightforward consequence of typical component sizes in an optical lab environment. We also assumed during the analysis that the overall bandwidth of the system is mainly restricted by the

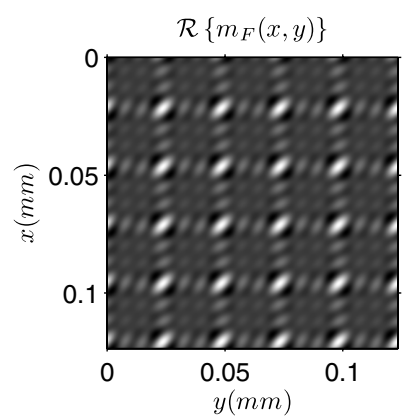

(a)

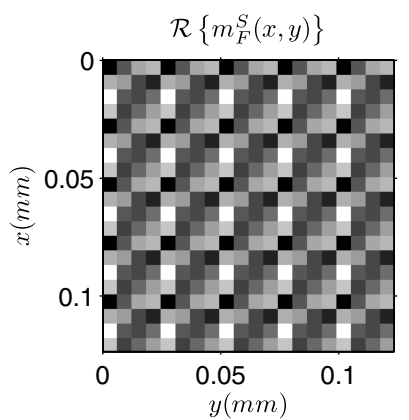

(b)
Fig. 10. (a) Fourier plane mask for the weights given in Eq. (11). (b) Pixellated Fourier plane mask that should be used for the weights given in Eq. (11). Both masks are displayed for five periods in each dimension, and only real parts are shown.
Fourier plane mask. This means that the lens apertures should not be too small, so that they do not cause a further restriction on the bandwidth. Under these conditions, Eqs. (17)-(21) provide a fairly accurate description of behavior of the physical setup.

Second, we should note that other optical implementations are also possible for the generic method proposed in Section 2. Assuming that we start with a single $P M \times Q N$ binary SLM, the critical issue is that, the binary SLM should be divided into $P Q$ sub-SLMs of size $M \times N$ and a properly weighted superposition of these sub-SLMs must be formed optically in a coherent manner. That superposition can be effectively formed using other optical components, such as beam splitters or prisms. However, in such options, each sub-SLM must be illuminated with a plane wave whose complex amplitude is equal to the corresponding weighting coefficient of that sub-SLM. Hence, a nonuniform illumination must be used for the binary SLM. In addition, since there are many sub-SLMs, we would need many beam splitters or prisms, whose physical dimensions must be suitable for placing side by side. All these factors complicate the implementation. But the presented $4 f$ system only requires the lenses and the Fourier and output plane masks. A common plane wave illumination is sufficient for the entire binary SLM. Then, the $4 f$ system automatically handles the mentioned properly weighted superposition of the subSLMs. Moreover, while creating the full-complex SLM, adjusting the widths of the Fourier plane mask, we can get rid of the diffraction orders of the SLM output. Hence, while not being the only possible option, we believe that the $4 f$ system is a convenient option.

Finally, let us discuss the main drawbacks of the proposed $4 f$ system. One of the significant drawbacks is that precise alignment is required between the optical components. For instance, if other components are perfectly placed but the Fourier plane mask is slightly off-positioned on the transverse plane, the sub-SLMs will be superposed with weights that are different than intended, and this will result in a malfunctioning of the system. However, we believe that easy test procedures can be developed to achieve the required precision in alignment in an optical environment.

Another drawback might be due to the light efficiency of the $4 f$ system. In practice, the input power (i.e., the power used to illuminate the binary SLM) will be partly lost as the light passes through the binary SLM, the lenses, and the masks, so that only a fraction of the input power will be delivered to the full-complex SLM and to the observation region. Actually, if we ignore the losses due to the binary SLM, the 
lenses, and the finite aperture size of the Fourier plane mask, and if we assume that the binary SLM pixels are independently distributed and for each pixel the values -1 or 1 are equally likely, a straightforward analysis yields that, on average, the fraction of the input power delivered to the full-complex SLM (i.e., the light efficiency of the system) is given by

$$
L_{\mathrm{eff}}=\frac{1}{P Q} \frac{1}{\eta^{2}} \sum_{p=0}^{P-1} \sum_{q=0}^{Q-1}|\bar{w}[p, q]|^{2},
$$

where $\eta$ is as given in Eq. (20). Hence, $L_{\text {eff }}$ depends on the selection for $\bar{w}[p, q]$. It can be shown that $L_{\text {eff }}$ varies between $\frac{1}{(P Q)^{2}}$ and $\frac{1}{P Q}$. For instance, for the $P=Q=4$ case, $L_{\text {eff }}$ changes between $0.39 \%$ and $6.25 \%$, and for the weights given in Eq. (11), it is about $1.08 \%$. For some applications, these efficiencies might be low. But as we pointed out in Section 2, for holographic purposes, even 4 bit quantization is usually sufficient $[\underline{4}, \underline{28}]$, so taking $P=2$ and $Q=2, L_{\text {eff }}$ can be made to vary between $12.5 \%$ and $25 \%$. We believe that, at least for $3 \mathrm{D}$ display purposes, this efficiency is sufficient, comparable to that of other schemes based on binary SLMs, and can be tolerated to enjoy the benefits of having a full-complex SLM.

\section{PIXELLATED AND QUANTIZED FOURIER PLANE MASKS}

The Fourier plane mask, denoted by $m_{F}(x, y)$ and given in Eq. (19), is the key component of the proposed $4 f$ system. This mask should be physically produced and placed in the Fourier plane. The problem is, the mask given in Eq. (19) is a continuous function of space coordinates taking on continuously varying gray values, so it is hard to physically produce. In this section, we consider the usage of pixellated and quantized Fourier plane masks, since such masks are easier to produce in practice.

Actually, the mask given in Eq. (19) is a continuous function of space coordinates because $H\left(\overline{\nu_{x}}, \nu_{y}\right)$ given in Eq. (7) is a continuous function of $\nu_{x}$ and $\nu_{y}$. Recall that $H\left(\nu_{x}, \nu_{y}\right)$ denotes the frequency response of the LSI system discussed in Subsection 3.A. Now, suppose instead of that system, we use another LSI system whose frequency response $H_{S}\left(\nu_{x}, \nu_{y}\right)$ is defined as:

$$
\begin{aligned}
H_{S}\left(\nu_{x}, \nu_{y}\right)= & \operatorname{rect}\left(\nu_{x} P M \Delta_{x}\right) \operatorname{rect}\left(\nu_{y} Q N \Delta_{y}\right) \\
& * *\left\{H ( \nu _ { x } , \nu _ { y } ) \sum _ { r = - \infty } ^ { \infty } \sum _ { t = - \infty } ^ { \infty } \delta \left(\nu_{x}-\frac{r}{P M \Delta_{x}},\right.\right. \\
& \left.\left.\nu_{y}-\frac{t}{Q N \Delta_{y}}\right)\right\} .
\end{aligned}
$$

As seen, $H_{S}\left(\nu_{x}, \nu_{y}\right)$ is obtained by sampling $H\left(\nu_{x}, \nu_{y}\right)$ and then applying zero-order interpolation on the resulting discrete signal. Recall that $H\left(\nu_{x}, \nu_{y}\right)$ is periodic with $\frac{1}{M \Delta_{x}}$ and $\frac{1}{N \Delta_{y}}$. Since it is sampled with sampling periods $\frac{1}{P M \Delta_{x}}$ and $\frac{1}{Q N \Delta_{y}}$, $H_{S}\left(\nu_{x}, \nu_{y}\right)$ is also periodic with $\frac{1}{M \Delta_{x}}$ and $\frac{1}{N \Delta_{y}}$. Because of zeroorder hold interpolation, $H_{S}\left(\nu_{x}, \nu_{y}\right)$ has a piecewise constant structure. Actually, this is the main reason for us to consider the new LSI system instead of the original LSI system; if we manage to create the full-complex SLM using the new LSI system, the new Fourier plane mask given as

$$
m_{F}^{S}(x, y)=\frac{1}{\eta} H_{B}\left(-\frac{x}{\lambda f},-\frac{y}{\lambda f}\right) H_{S}\left(-\frac{x}{\lambda f},-\frac{y}{\lambda f}\right)
$$

will become a pixellated mask. However, we should first show that we can also create the full-complex SLM using the new LSI system.

Taking the inverse Fourier transform of $H_{S}\left(\nu_{x}, \nu_{y}\right)$, we see that the impulse response of the new LSI system is given as

$$
\begin{aligned}
h_{S}(x, y)= & \operatorname{sinc}\left(\frac{x}{P M \Delta_{x}}\right) \operatorname{sinc}\left(\frac{y}{Q N \Delta_{y}}\right) \\
& \times \sum_{r=-\infty}^{\infty} \sum_{t=-\infty}^{\infty} h\left(x-r P M \Delta_{x}, y-t Q N \Delta_{y}\right),
\end{aligned}
$$

where $h(x, y)$ denotes the impulse response of the original LSI system [see Eq. (6)]. As seen, sampling of $H\left(\nu_{x}, \nu_{y}\right)$ causes a periodic replication of $h(x, y)$ in space where the replicas are spaced by $P M \Delta_{x}$ and $Q N \Delta_{y}$, and zero-order hold interpolation creates the sinc roll-off factor. Similar to $h(x, y), h_{S}(x, y)$ consists of impulses that are spaced by $M \Delta_{x}$ and $N \Delta_{y}$, but unlike $h(x, y)$, the number of impulses in $h_{S}(x, y)$ is infinite. Similar to Subsection 3.A, let $g_{S}(x, y)=b(x, y) * * h_{S}(x, y) \quad$ and $\quad s_{S}(x, y)=g_{S}(\overline{x, y})$ $\operatorname{rect}\left(\frac{x-(P-0.5) M \Delta_{x}}{M \Delta_{x}}\right) \operatorname{rect}\left(\frac{y-(Q-0.5) N \Delta_{y}}{N \Delta_{y}}\right)$. It is easy to show that

$$
s_{S}(x, y)=\sum_{p=0}^{P-1} \sum_{q=0}^{Q-1} \bar{w}^{\prime}[P-1-p, Q-1-q] b_{p, q}\left(x-x_{0}, y-y_{0}\right)
$$

where $x_{0}=(P-1) M \Delta_{x}, y_{0}=(Q-1) N \Delta_{y}$, and

$$
\bar{w}^{\prime}[p, q]=\bar{w}[p, q] \operatorname{sinc}\left(\frac{p}{P}\right) \operatorname{sinc}\left(\frac{q}{Q}\right)
$$

for $0 \leq p \leq P-1$ and $0 \leq q \leq Q-1$. We see upon comparison of Eq. (26) with Eq. (10) that, when we use the new LSI system, the only change is that, when forming $s_{S}(x, y)$, sub-SLMs are weighted by $\bar{w}^{\prime}[p, q]$ instead of $\bar{w}[p, q]$. The main reason for this change is the zero-order hold interpolation that is used when obtaining $H_{S}\left(\nu_{x}, \nu_{y}\right)$ from $H\left(\nu_{x}, \nu_{y}\right)$. However, this change does not create any problem. In particular, now we should specify $\bar{w}^{\prime}[p, q]$ rather than specifying $\bar{w}[p, q]$. After specifying $\bar{w}^{\prime}[p, q]$, we should find $\bar{w}[p, q]$ according to Eq. (27), and then we should design the new LSI system according to Eqs. (7) and (23). When this is done, the new LSI system will produce the same output as the original LSI system (i.e., $s_{S}(x, y)=s(x, y)$ ), implying that instead of the old Fourier plane mask given in Eq. (19), we can use the mask given in Eq. (24). This new mask, which has a pixellated structure, is also periodic in its spatial support with periods $\frac{\lambda f}{M \Delta_{x}}$ and $\frac{\lambda f}{N \Delta_{y}}$. The pixel widths of the new mask are given by $\frac{\lambda f}{P M \Delta_{x}}$ and $\frac{\lambda f}{Q N \Delta_{y}}$. Therefore, in each period of the mask, there are $P \times Q$ pixels. Note that, since the mask widths must be greater than $\frac{\lambda f}{\Delta_{x}}$ and $\frac{\lambda f}{\Delta_{y}}$ by Eq. (16), the new mask should have at least $P M \times Q N$ pixels.

If the physical production process only dictates that the Fourier plane mask should be pixellated, but no quantization 
on pixel values is required, given $\bar{w}^{\prime}[p, q]$, we only need to compute $\bar{w}[p, q], H\left(\nu_{x}, \nu_{y}\right)$, and $H_{S}\left(\nu_{x}, \nu_{y}\right)$ as explained earlier, and prepare the pixellated Fourier plane mask $m_{F}^{S}(x, y)$ according to Eq. (24). For instance, for the $P=Q=4$ case, if we want $\bar{w}^{\prime}[p, q]$ to be equal to the weights given in Eq. (11), the pixellated mask shown in Fig. 10(b) should be used. The mask in Fig. 10(b) produces the same output (possibly up to a constant amplitude factor) with the mask in Fig. 10(a).

In practice, usually, there is also a quantization constraint on the pixel values of the Fourier plane mask. In such cases, the correct approach is to take the Fourier plane mask as given and determine the implied $\bar{w}^{\prime}[p, q]$. Given $m_{F}^{S}(x, y)$, using simple Fourier transform relations, it can be shown that the implied $\bar{w}^{\prime}[p, q]$ becomes

$$
\begin{aligned}
\bar{w}^{\prime}[p, q]= & \frac{1}{16} \operatorname{sinc}\left(\frac{p}{P}\right) \operatorname{sinc}\left(\frac{q}{Q}\right) \\
& \times \sum_{r=0}^{P-1} \sum_{t=0}^{Q-1} m_{F}^{S}\left(\frac{r \lambda f}{P M \Delta_{x}}, \frac{t \lambda f}{Q N \Delta_{y}}\right) e^{j 2 \pi\left(\frac{p r}{P}+\frac{q t}{Q}\right)}
\end{aligned}
$$

for $0 \leq p \leq P-1$ and $0 \leq q \leq Q-1$. Note that, in the above equation, $m_{F}^{S}\left(\frac{r \lambda f}{P M \Delta_{x}}, \frac{t \lambda f}{Q N \Delta_{y}}\right)$ denotes the pixel values of the Fourier plane mask. In the presence of a quantization constraint, Fourier plane masks should be designed according to the above equation. In particular, we should first assign the values of the pixels of the mask taking into account the quantization constraint on them. Then, we should compute the implied weights according to the above equation, and then we should compute the complex values achievable by a pixel of the new SLM based on these weights. If these values are few in number or have poor coverage of the complex plane, we should redesign the mask.

Assuming $P=Q=4$, we go through a number of examples and show that even Fourier plane masks with quite limited pixel values can lead to $\bar{w}^{\prime}[p, q]$ that generate a large number of complex values for the pixels of the new SLM. Figure 11(a)

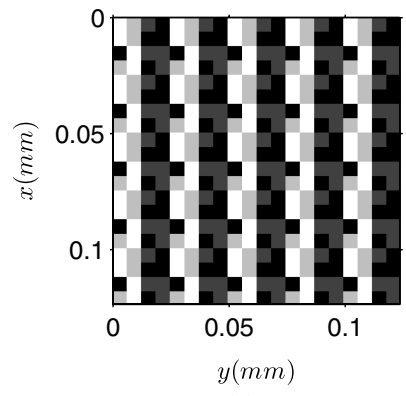

(a)

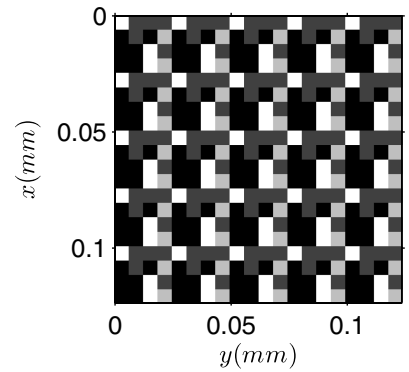

(c)

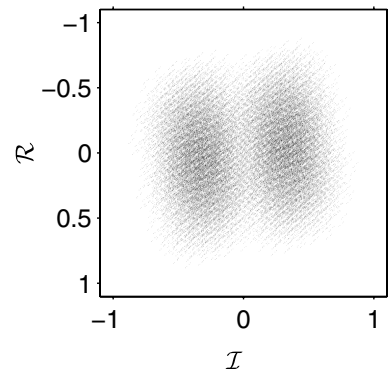

(b)

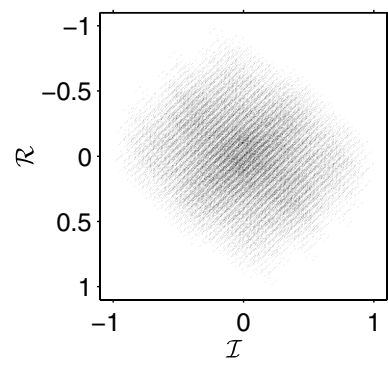

(d)
Fig. 11. (a), (c) Four-level Fourier plane masks. (b), (d) Achievable complex numbers. illustrates a mask whose pixels are equal to \pm 1 or $\pm j$. Hence, there are only four levels available for a pixel of the mask. Figure 11(b) illustrates the complex numbers available for a pixel of the new SLM when we use this mask. There are $2^{16}$ different complex numbers on this figure. Another four-level example is illustrated in Figs. 11(c) and 11(d). The number of achievable complex numbers is again $2^{16}$. [In Figs. 11(a) and $11(\mathrm{c})$, white, light gray, dark gray, and black pixels respec$\overline{\text { tively }}$ have values $1, j,-j$, and -1 .] Therefore, even using the simple masks illustrated in Fig. 11, we can produce the new full-complex SLM without any loss in the information content. Moreover, the achievable complex numbers have a good coverage of the complex plane. Even simpler masks can be used if we accept a slight degradation in this coverage. Figures 12(a) and 12(c) illustrate two masks whose pixels are equal to \pm 1 or 0 . Hence, there are only three levels available for a pixel of the mask. Figures $\underline{12(b)}$ and $12(\mathrm{~d})$ illustrate the resulting complex numbers that can be achieved. There are again $2^{16}$ different complex numbers in both figures, but their coverage of the complex plane is slightly worse than the four-level examples. [In Figs. 12(a) and 12(c), white, gray, and black pixels respectively have values $\overline{1,0}$, and -1.) Yet even simpler masks can be used if we accept to achieve a reduced number of complex numbers (i.e., if we tolerate some loss in the information content). Figures 13(a) and 13(c) illustrate two binary masks whose pixels are equal to \pm 1 . Figures $\underline{13(b)}$ and $\underline{13(d)}$ illustrate the resulting complex numbers that can be achieved. This time, there are only $2^{15}$ different complex numbers in both figures (implying that the full-complex SLM is 15 bit, so 1 bit of information is lost per pixel), which is lower than $2^{16}$ but still high, and the coverage of the complex plane is acceptable. [In Figs. 13(a) and 13(c), white and black pixels respectively have values 1 and -1 .] The 1 bit per pixel loss in the information content may be tolerated for the convenience of using binary masks, which are quite

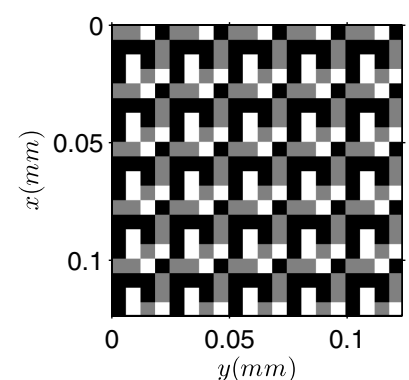

(a)

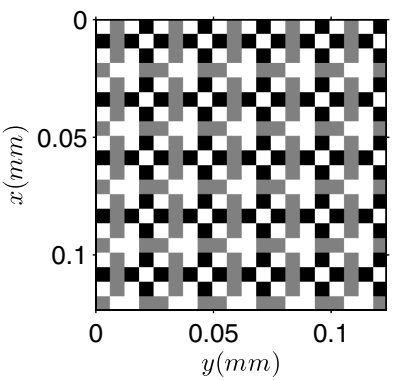

(c)

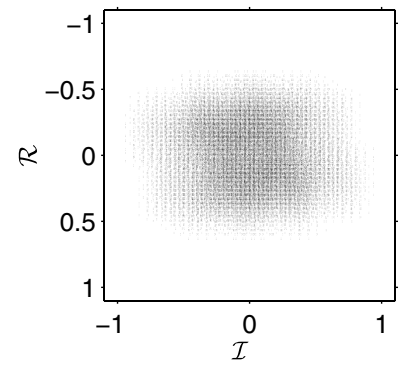

(b)

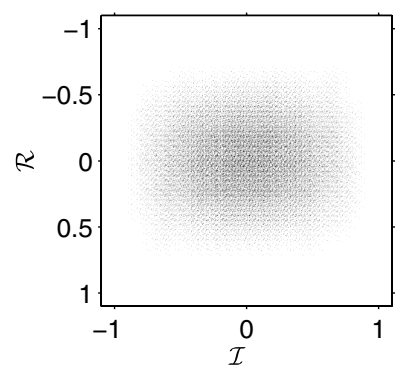

(d)
Fig. 12. (a), (c) Three-level Fourier plane masks. (b), (d) Achievable complex numbers. 


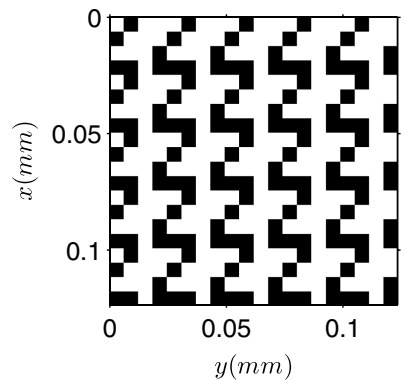

(a)

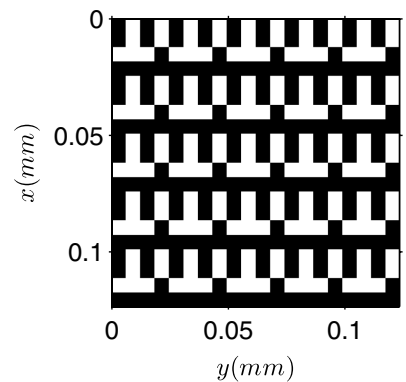

(c)

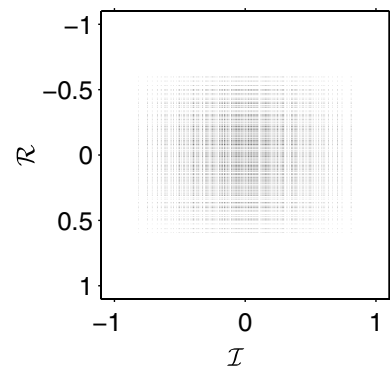

(b)

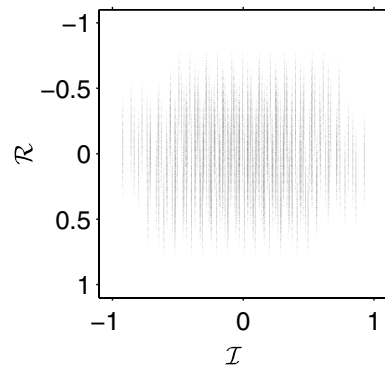

(d)
Fig. 13. (a), (c) Binary Fourier plane masks. (b), (d) Achievable complex numbers.

easy to physically produce. These examples show that as the quantization constraint on the Fourier plane mask gets harsher, the number of available complex values for a pixel of the SLM can decrease and the coverage of the complex plane can get worse. However, since the number of available levels is still large, given a typical desired full-complex SLM pattern, the quantization error will be still quite low (though it may increase slightly) and no noticeable degradation in final reconstruction quality will take place.

To sum up, Eqs. (26) and (27) indicate that, instead of any continuous Fourier plane mask, we can design and use an equivalent pixellated mask and get the same output. The examples in Figs. 11-13 indicate that, even in the case of a severe quantization constraint, it is possible to design Fourier plane masks such that the complex values that are available for a pixel of the full-complex SLM are large in number and have a good coverage of the complex plane. Therefore, pixellation and quantization of the Fourier plane mask do not cause any noticeable degradation in the system performance in terms of reconstruction quality. However, in the case of a pixellated mask, the light efficiency will be slightly decreased relative to the continuous mask case. This is because of the fact that the pixellated mask will cause the emergence of higher-order waves, which divert some of the input power. These waves travel in high angles and are blocked at the output plane mask stage, causing a smaller portion of the input power to be delivered to the full-complex SLM and thus to the observation region. Roughly, on average, the efficiency will be decreased by about $20 \%$ at this stage relative to the continuous mask case. This decrease can be minimized if phase-only Fourier plane masks, such as the ones shown in Figs. 11 and 13, are used. We assume that this additional loss can be tolerated for the convenience of using pixellated and quantized Fourier plane masks.

\section{CONCLUSION}

In this paper, we first proposed a generic method for effectively creating full-complex SLMs out of binary SLMs. The method relies on forming a properly weighted superposition of binary SLMs. We showed that, in this manner, informationwise, binary SLMs are utilized in the most efficient manner. Then, we proposed a $4 f$ system as a possible optical implementation of our generic method. In addition to forming the full-complex SLM, this $4 f$ setup also enables us to get rid of the disturbing higher diffraction orders of the SLM output. We showed that the parameters and components of the system can easily be customized for different production technologies. One main drawback of the system is the precise alignment requirement, but we believe that easy-to-apply optical test procedures can be designed to satisfy it. Another drawback may be due to light efficiency, but we assume that, in 3D display applications, the levels are tolerable. Compared to previous approaches, the most important feature of our approach is that we tried to use the full potential of the binary SLMs when creating the full-complex SLMs. Actually, our generic method can be tailored to create full-complex SLMs out of multilevel amplitude-only or phase-only SLMs. In this case, less complicated optical systems can be used for optical implementation. However, we believe that the robust behavior of binary SLMs justify our choice for selecting them to create the full-complex SLMs. We believe that, for commercially available binary SLMs, the proposed $4 f$ system can be implemented within a small volume. Therefore, multiple replicas of the $4 f$ system can be conveniently placed side by side to create full-complex SLM arrays to be used in applications such as $3 \mathrm{D}$ displays.

\section{ACKNOWLEDGMENTS}

This work was partially supported by the European Commission within FP6 under grant 511568 with acronym 3DTV. E. Ulusoy acknowledges partial support of the Scientific and Technological Research Council of Turkey. H. M. Ozaktas acknowledges partial support of the Turkish Academy of Sciences.

\section{REFERENCES}

1. C. Slinger, C. Cameron, and M. Stanley, "Computer-generated holography as a generic display technology," Computer 38, 46-53 (2005).

2. H. M. Ozaktas and L. Onural, eds., Three-Dimensional Television: Capture, Transmission, Display, Series in Signals and Communication Technology (Springer, 2008).

3. F. Yaras, H. Kang, and L. Onural, "State of the art in holographic displays: a survey,” J. Disp. Technol. 6, 443-454 (2010).

4. L. Onural, F. Yaras, and H. Kang, "Digital holographic threedimensional video displays,” Proc. IEEE 99, 576-589 (2011).

5. L. Onural, A. Gotchev, H. M. Ozaktas, and E. Stoykova, "A survey of signal processing problems and tools in holographic threedimensional television," IEEE Trans. Circuits Syst. Video Technol. 17, 1631-1646 (2007).

6. L. Onural and H. M. Ozaktas, "Signal processing issues in diffraction and holographic 3DTV," Signal Process., Image Commun. 22, 169-177 (2007).

7. R. W. Cohn, "Pseudorandom encoding of complex-valued functions onto amplitude-coupled phase modulators," J. Opt. Soc. Am. A 15, 868-883 (1998).

8. R. W. Cohn, "Analyzing the encoding range of amplitude-phase coupled spatial light modulators," Opt. Eng. 38, 361-367 (1999). 
9. Y. Yang, H. Stark, D. Gurkan, C. L. Lawson, and R. W. Cohn, "High-diffraction-efficiency pseudorandom encoding," J. Opt. Soc. Am. A 17, 285-293 (2000).

10. C. Stolz, L. Bigue, and P. Ambs, "Implementation of highresolution diffractive optical elements on coupled phase and amplitude spatial light modulators," Appl. Opt. 40, 6415-6424 (2001).

11. V. Arrizon, "Optimum on-axis computer-generated hologram encoded into low-resolution phase-modulation devices," Opt. Lett. 28, 2521-2523 (2003).

12. J. A. Davis, K. O. Valadez, and D. M. Cottrell, "Encoding amplitude and phase information onto a binary phase-only spatial light modulator," Appl. Opt. 42, 2003-2008 (2003).

13. V. Arrizon, G. Mendez, and D. S. de La-Llave, "Accurate encoding of arbitrary complex fields with amplitude-only liquid crystal spatial light modulators," Opt. Express 13, 7913-7927 (2005).

14. V. Arrizon, U. Ruiz, R. Carrada, and L. A. Gonzalez, "Pixelated phase computer holograms for the accurate encoding of scalar complex fields," J. Opt. Soc. Am. A 24, 3500-3507 (2007).

15. E. Ulusoy, L. Onural, and H. M. Ozaktas, "Synthesis of threedimensional light fields with binary spatial light modulators," J. Opt. Soc. Am. A 28, 1211-1223 (2011).

16. R. Tudela, I. Labastida, E. Martin-Badosa, S. Vallmitjana, I. Juvells, and A. Carnicer, "A simple method for displaying fresnel holograms on liquid crystal panels," Opt. Commun. 214, 107-114 (2002).

17. R. Tudela, E. Martin-Badosa, I. Labastida, S. Vallmitjana, I. Juvells, and A. Carnicer, "Wavefront reconstruction by adding modulation capabilities of two liquid crystal devices," Opt. Eng. 43, 2650-2657 (2004).

18. D. A. Gregory, J. C. Kirsch, and E. C. Tam, "Full complex modulation using liquid-crystal televisions," Appl. Opt. 31, 163-165 (1992).
19. R. D. Juday, "Full-complex modulation with two one-parameter spatial light modulators," U.S. patent 5,416,618 (1995).

20. L. G. Neto, D. Roberge, and Y. Sheng, "Full-range, continuous, complex modulation by the use of two coupled-mode liquidcrystal televisions," Appl. Opt. 35, 4567-4576 (1996).

21. P. M. Birch, R. Young, D. Budgett, and C. Chatwin, "Two-pixel computer-generated hologram with a zero-twist nematic liquidcrystal spatial light modulator," Opt. Lett. 25, 1013-1015 (2000).

22. V. Arrizon, "Complex modulation with a twisted-nematic liquidcrystal spatial light modulator: double-pixel approach," Opt. Lett. 28, 1359-1361 (2003).

23. E. G. van Putten, I. M. Vellekoop, and A. P. Mosk, "Spatial amplitude and phase modulation using commercial twisted nematic LCDs," Appl. Opt. 47, 2076-2081 (2008).

24. E. Ulusoy, L. Onural, and H. M. Ozaktas, "Signal processing for three-dimensional holographic television displays that use binary spatial light modulators," in Proceedings of IEEE Conference on Signal Processing and Communications Applications Conference (IEEE, 2010), pp. 41-44, in Turkish.

25. T. Kreis, P. Aswendt, and R. Hofling, "Hologram reconstruction using a digital micromirror device," Opt. Eng. 40, 926-933 (2001).

26. D. Dudley, W. Duncan, and J. Slaughter, "Emerging digital micromirror device (DMD) applications," white paper (Texas Instruments, 2003).

27. R. C. Gonzales and R. E. Woods, Digital Image Processing (Prentice-Hall, 2002).

28. T. J. Naughton, Y. Frauel, B. Javidi, and E. Tajahuerce, "Compression of digital holograms for three-dimensional object reconstruction and recognition," Appl. Opt. 41, 4124-4132 (2002).

29. L. Onural, "Sampling of the diffraction field," Appl. Opt. 39, 5929-5935 (2000).

30. L. Onural, "Exact analysis of the effects of sampling of the scalar diffraction field," J. Opt. Soc. Am. A 24, 359-367 (2007).

31. B. E. A. Saleh and M. C. Teich, Fundamentals of Photonics, 2nd ed. (Wiley, 2007). 